Pacific Journal of Mathematics

SUFFICIENT CONDITIONS FOR A RIEMANNIAN MANIFOLD 


\title{
SUFFICIENT CONDITIONS FOR A RIEMANNIAN MANIFOLD TO BE LOCALLY SYMMETRIC
}

\author{
Kouei Sekigawa and ShÛkichi Tanno
}

In a locally symmetric Riemannian manifold the scalar curvature is constant and each $k$-th covariant derivative of the Riemannian curvature tensor vanishes. In this note, we show that if the covariant derivatives of the Riemannian curvature tensor satisfy some algebraic conditions at each point, then the Riemannian manifold is locally symmetric.

Let $R$ be the Riemannian curvature tensor of a Riemannian manifold $M^{m}$ with a positive-definite metric tensor $g$. Manifolds and tensors are assumed to be of class $C^{\infty}$ unless otherwise stated. We denote by $\nabla$ the Riemannian connection defined by $g$. For tangent vectors $X$ and $Y$, we consider $R(X, Y)$ as a derivation of the tensor algebra at each point. A conjecture by $K$. Nomizu [4] is that $R(X, Y) \cdot R=0$ on a complete and irreducible manifold $M^{m}(m \geqq 3)$ implies $\nabla R=0$, that is, $M^{m}$ is locally symmetric. Here we consider some additional conditions.

For an integer $k$ and tangent vectors $V_{k}, \cdots, V_{1}$ at a point $p$ of $M^{m}$, we adopt a notation:

$$
\begin{aligned}
\left(\nabla_{V}^{k} R\right) & =\left(V_{k}, V_{k-1}, \cdots, V_{1} ; \nabla^{k} R\right) \\
& =\left(V_{k}^{t} V_{k-1}^{s} \cdots V_{1}^{r} \nabla_{t} \nabla_{s} \cdots \nabla_{r} R_{b c d}^{a}\right),
\end{aligned}
$$

where $V_{k}^{t}$, etc., are components of $V_{k}$, etc., and $\nabla_{t} \nabla_{s} \cdots \nabla_{r} R_{b c d}^{a}$ are components of the $k$-th covariant derivative $\nabla^{k} R$ of $R$ in local coordinates.

Proposition 1. Let $M^{m}(m \geqq 3)$ be a real analytic Riemannian manifold. Assume that

(1.0) the restricted holonomy group is irreducible,

(1.1) $R(X, Y) \cdot R=0$,

(1.2) $R(X, Y) \cdot\left(\nabla_{V}^{k} R\right)=0$ for $k=1,2, \cdots$.

Then $M^{m}$ is locally symmetric.

Here we note that condition (1.0) means that it holds at some, hence every, point and condition (1.1), and (1.2), mean that for any point $p$ and for any tangent vectors $X, Y, V_{k}, \cdots, V_{1}$ at $p$, they hold.

Proposition 2. Let $M^{m}(m \geqq 3)$ be a Riemannian manifold. Assume (1.1) and (1.2) and that 
(1.0)' the infinitesimal holonomy group is irreducible at every point. Then $M^{m}$ is locally symmetric.

Propositions 1 and 2 are essentially related to the following results.

Proposition 3. Let $M^{m}(m \geqq 3)$ be a Riemannian manifold. Assume that the restricted holonomy group $H^{0}$ (the infinitesimal holonomy group $H^{\prime}$, resp.) is irreducible, and $R$ is invariant by $H^{0}$ $\left(H^{\prime}\right.$, resp.). Then $M^{m}$ is locally symmetric.

Proposition 3'. (J. Simons [5], p. 233) Let $M^{m}(m \geqq 3)$ be an irreducible Riemannian manifold. Assume that $R$ is invariant by the holonomy group $H$. Then $M^{m}$ is locally symmetric.

Proposition 3 is a generalization of a result by A. Lichnerowicz ([2], p. 11), which contains an assumption of compactness. We remark here that condition (1.2) is equivalent to

$$
R(X, Y) \cdot\left(\nabla_{V_{k}} \nabla_{V_{k-1}} \cdots \nabla_{V_{1}} R\right)=0 \text { for } k=1,2, \cdots,
$$

where $X, Y, V_{k}, \cdots, V_{1}$ are vector fields on $M^{m}$.

With respect to Nomizu's conjecture and the above propositions we have

THEOREM 4. Let $M^{m}(m \geqq 3)$ be a Riemannian manifold. Assume that

(i) the scalar curvature $S$ is constant,

(ii) $R(X, Y) \cdot R=0$,

(iii) $R(X, Y) \cdot \nabla_{V} R=0$,

(iv) $R(X, Y) \cdot\left(X, V ; \nabla^{2} R\right)=0$,

(or (iv) $R(X, Y) \cdot \nabla_{X} \nabla_{V} R=0$ for vector fields).

Then $M^{m}$ is locally symmetric.

Theorem 5. Let $M^{m}(m \geqq 3)$ be a Riemannian manifold. Assume that

(i) the Ricci curvature tensor $R_{1}$ is parallel; $\nabla R_{1}=0$,

(ii) $R(X, Y) \cdot R=0$,

(iii) $R(X, Y) \cdot \nabla_{V} R=0$.

Then $M^{m}$ is locally symmetric.

In Theorems 4 and 5 , if $m=2$, then $\nabla R_{1}=0$ implies $\nabla R=0$.

In Theorem 5, if $M^{m}$ is compact, (iii) can be dropped (A. Lichnerowicz [2], or K. Yano [6], p. 222).

In $\S 2$ we reduce proofs of Propositions 1 and 2 to that of Proposi- 
tion 3, and next we reduce proofs of Propositions 3 and $3^{\prime}$ to that of Theorem 4. In $\S 3$ we prove Theorems 4 and 5 .

2. Holonomy algebras. Conditions (1.1) and (1.2) imply that

$$
\begin{aligned}
{\left[R(X, Y),\left(\nabla_{V}^{k} R\right)(A, B)\right]=} & \left(\nabla_{V}^{k} R\right)(R(X, Y) A, B) \\
& +\left(\nabla_{V}^{k} R\right)(A, R(X, Y) B)
\end{aligned}
$$

for $k=0,1, \cdots$, where $\nabla^{0} R$ means $R$, and $\left[T, T^{\prime}\right]$ for linear transformations $T, T^{\prime}$ means $T T^{\prime}-T^{\prime} T$.

Now we show

LEMMA 2.1. The condition (2.1) implies

$$
\begin{aligned}
{\left[\left(\nabla_{W}^{j} R\right)(X, Y),\left(\nabla_{V}^{k} R\right)(A, B)\right]=} & \left(\nabla_{V}^{k} R\right)\left(\left(\nabla_{W}^{j} R\right)(X, Y) A, B\right) \\
& +\left(\nabla_{V}^{k} R\right)\left(A,\left(\nabla_{W}^{j} R\right)(X, Y) B\right)
\end{aligned}
$$

for $j, k=0,1,2, \cdots$ And (2.1) is equivalent to

$$
\begin{aligned}
{\left[\left(\nabla_{W}^{j} R\right)(X, Y), R(A, B)\right]=} & R\left(\left(\nabla_{W}^{j} R\right)(X, Y) A, B\right) \\
& +R\left(A,\left(\nabla_{W}^{j} R\right)(X, Y) B\right)
\end{aligned}
$$

for $j=0,1,2, \cdots$.

Proof. We prove (2.2) by induction in $j$ and by tensor calculus in local coordinates. By (2.1), (2.2) holds for $(j, k)=(0, k), k=0$, $1,2, \cdots$ Assume that (2.2) holds for $(j-1, k),(j-2, k), \cdots,(0, k)$, $k=0,1,2, \cdots$. Then, denoting by $\nabla_{t} \nabla_{s} \cdots \nabla_{r} R_{u x y}^{p}$ the $j$-th covariant derivative of $R$ and by $\nabla_{f} \cdots \nabla_{e} R_{q a b}^{u}$ the $k$-th covariant derivative of $R$, we show

$$
\begin{aligned}
& \nabla_{t} \nabla_{s} \cdots \nabla_{r} R_{u x y}^{p} \nabla_{f} \cdots \nabla_{e} R_{q a b}^{u}-\nabla_{f} \cdots \nabla_{e} R_{u a b}^{p} \nabla_{t} \nabla_{s} \cdots \nabla_{r} R_{q x y}^{u} \\
& =\nabla_{f} \cdots \nabla_{e} R_{q v b}^{p} \nabla_{t} \nabla_{s} \cdots \nabla_{r} R_{a x y}^{v}+\nabla_{f} \cdots \nabla_{e} R_{q a v}^{p} \nabla_{t} \nabla_{s} \cdots \nabla_{r} R_{b x y}^{v}
\end{aligned}
$$

In fact, we have

$$
\begin{aligned}
\nabla_{t} \nabla_{s} & \cdots \nabla_{r} R_{u x y}^{p} \nabla_{f} \cdots \nabla_{e} R_{q a b}^{u}-\nabla_{f} \cdots \nabla_{e} R_{u a b}^{p} \nabla_{t} \nabla_{s} \cdots \nabla_{r} R_{q x y}^{u} \\
= & \nabla_{t}\left(\nabla_{s} \cdots \nabla_{r} R_{u x y}^{p} \nabla_{f} \cdots \nabla_{e} R_{q a b}^{u}\right) \\
& -\nabla_{s} \cdots \nabla_{r} R_{u x y}^{p} \nabla_{t} \nabla_{f} \cdots \nabla_{e} R_{q a b}^{u} \\
& -\nabla_{t}\left(\nabla_{f} \cdots \nabla_{e} R_{u a b}^{p} \nabla_{s} \cdots \nabla_{r} R_{q x y}^{u}\right) \\
& +\nabla_{t} \nabla_{f} \cdots \nabla_{e} R_{u a b}^{p} \nabla_{s} \cdots \nabla_{r} R_{q x y}^{u} \\
= & \nabla_{t}\left(\nabla_{f} \cdots \nabla_{e} R_{q u b}^{p} \nabla_{s} \cdots \nabla_{r} R_{a x y}^{u}+\nabla_{f} \cdots \nabla_{e} R_{q a v}^{p} \nabla_{s} \cdots \nabla_{r} R_{b x y}^{v}\right) \\
& -\nabla_{s} \cdots \nabla_{r} R_{u x y}^{p} \nabla_{t} \nabla_{f} \cdots \nabla_{e} R_{q a b}^{u} \\
& +\nabla_{t} \nabla_{f} \cdots \nabla_{e} R_{u a b}^{p} \nabla_{s} \cdots \nabla_{r} R_{q x y}^{u} \quad(\text { by }(2.2) \text { for }(j-1, k)) \\
= & \nabla_{f} \cdots \nabla_{e} R_{q v b}^{p} \nabla_{t} \nabla_{s} \cdots \nabla_{r} R_{a x y}^{v}+\nabla_{f} \cdots \nabla_{e} R_{q a v}^{p} \nabla_{t} \nabla_{s} \cdots \nabla_{r} R_{b x y}^{v}
\end{aligned}
$$




$$
\begin{aligned}
& +\left(\nabla_{t} \nabla_{f} \cdots \nabla_{e} R_{u a b}^{p} \nabla_{s} \cdots \nabla_{r} R_{q x y}^{u}-\nabla_{s} \cdots \nabla_{r} R_{u x y}^{p} \nabla_{t} \nabla_{f} \cdots \nabla_{e} R_{q a b}^{u}\right) \\
& +\left(\nabla_{t} \nabla_{f} \cdots \nabla_{e} R_{q u b}^{p} \nabla_{s} \cdots \nabla_{r} R_{a x y}^{v}+\nabla_{t} \nabla_{f} \cdots \nabla_{e} R_{q a v}^{p} \nabla_{s} \cdots \nabla_{r} R_{b x y}^{v}\right) .
\end{aligned}
$$

The second and third terms vanish by $(2.2)$ for $(j-1, k+1)$. Therefore we have (2.4).

Similarly we can show that (2.3) implies (2.2), including (2.1).

By the theory of holonomy groups (cf. A. Nijenhuis [3]), the set of linear transformations

$$
R(X, Y),\left(\nabla_{W} R\right)(X, Y),\left(\nabla_{W}^{2} R\right)(X, Y), \cdots
$$

for $X, Y, W_{1}, \cdots \in M_{p}$, the tangent space to $M$ at $p$ of $M$, spans a Lie algebra $h_{p}^{\prime}$ called the infinitesimal holonomy algebra at $p . h_{p}^{\prime}$ generates the infinitesimal holonomy group $H_{p}^{\prime}$ which is a subgroup of the local holonomy group $H_{p}^{*}=H_{p}^{0}(U)$. Clearly $H_{p}^{*}$ is a subgroup of the restricted holonomy group $H_{p}^{0}$. If a Riemannian manifold is real analytic we have $H^{\prime}=H^{*}=H^{0}$.

The condition (2.3) implies that

$$
[T, R(A, B)]=R(T A, B)+R(A, T B)
$$

for any $T \in h_{p}^{\prime}$. This says that $R$ is invariant by $T$. Therefore, for any element $\alpha \in H_{p}^{\prime}$ we have

$$
\alpha R(A, B) C=R(\alpha A, \alpha B) \alpha C \quad \text { for } A, B, C \in M_{p} \text {. }
$$

Thus, we have reduced proofs of Propositions 1 and 2 to proof of Proposition 3.

Since (2.7) or (2.6) is equivalent to (2.1), condition (2.7) implies conditions (ii), (iii) and (iv) of Theorem 4. Consequently, if we show that, under the conditions in Proposition 3 ( $3^{\prime}$, resp.), the scalar curvature $S$ is constant, then Proposition 3 ( $3^{\prime}$, resp.) will follow from Theorem 4.

Let $E_{i}, 1 \leqq i \leqq m$, be an orthonormal basis at $p$. Then the Ricci curvature tensor $R_{1}$ is given by

$$
R_{1}(X, Y)=\sum_{i} g\left(R\left(X, E_{i}\right) Y, E_{i}\right) .
$$

Since $R$ is invariant by $H^{\prime}$ or $H^{0}$ or $H$, we have $R_{1}(X, Y)=R_{1}(\alpha X, \alpha Y)$ for any $\alpha \in H^{\prime}$, or $H^{0}$ or $H$. Since $H^{\prime}$ or $H^{0}$ or $H$ is irreducible, we have some real number $\lambda$ so that $R_{1}=\lambda g$ at $p$. Because $p$ is an arbitrary point of $M$ and $m \geqq 3, \lambda$ is constant on $M$, and hence $S=$ $m \lambda$ is constant.

3. Proofs of Theorems 4 and 5. To prove theorems it suffices to show two propositions below. 
Proposition 3.1. On $M^{m}(m \geqq 3)$ assume that

(i) the scalar curvature $S$ is constant,

(ii) $(R(X, Y) \cdot R)(X, V)=0$,

(iii) $\left(R(X, Y) \cdot \nabla_{V} R\right)(X, Y) V=0$,

(iv) $\left(R(X, Y) \cdot \nabla_{V} R_{1}\right)(V, X)=0$,

(v) $\left(R(X, Y) \cdot\left(X, V ; \nabla^{2} R_{1}\right)(V, Y)=0\right.$,

(or $(\mathrm{v})^{\prime}\left(R(X, Y) \cdot \nabla_{X} \nabla_{V} R_{1}\right)(V, Y)=0$ for vector fields).

Then we have $\nabla R=0$.

Proof. Let $\left\{E_{i}\right\}$ be an orthonormal basis at $p$ of $M$. Put $X=E_{x}$, $Y=E_{y}, V=E_{v}$ in (iii) and take a sum on $x, y, v$. Then we have

$$
R^{i r x y} \nabla_{v} R_{r x y}^{v}-R^{r v x y} \nabla_{v} R_{r x y}^{i}-R_{x y}^{r x} \nabla_{v} R_{r}^{i v}{ }_{r}^{y}-R_{x y}^{r y} \nabla_{v} R_{r}^{i v x}=0 \text {. }
$$

The third and fourth terms vanish. We apply the second Bianchi identity to the first two terms;

$$
\begin{aligned}
R^{i r x y}\left(-\nabla_{x} R_{r y v}^{v}-\nabla_{y} R_{r}{ }^{v}{ }_{v x}\right)= & -2 R^{i r x y} \nabla_{y} R_{r x}, \\
-R^{r v x y}\left(-\nabla_{i} R_{r v x y}-\nabla_{r} R_{v i x y}\right)= & R^{r v x y} \nabla_{i} R_{r v x y}+R^{r v x y} \nabla_{r} R_{v i x y} \\
= & R^{r v x y} \nabla_{i} R_{r v x y}+R^{r v x y} \nabla_{v} R_{i r x y} .
\end{aligned}
$$

Therefore, we have

$$
-4 R^{i r x y} \nabla_{y} R_{r x}+R^{r v x y} \nabla^{i} R_{r v x y}=0 .
$$

Likewise, (iv) implies that

$$
R_{y}^{r v x} \nabla_{v} R_{r x}+R_{x y}^{r x} \nabla_{v} R_{r}^{v}=0 .
$$

And (v) implies that

$$
R^{r x y} \nabla_{x} \nabla_{v} R_{r y}+R_{y}^{r}{ }^{x y} \nabla_{x} \nabla_{v} R_{r}^{v}=0 .
$$

For $(\mathrm{v})^{\prime}$ we assume that $E_{i}$ are local vector fields such that $\left(\nabla E_{i}\right)_{p}=0$ and $\left\{E_{i}\right\}$ forms an orthonormal basis at $p$. Then we have the same (3.3).

Since $\nabla_{v} R_{r}^{v}=(1 / 2) \nabla_{r} S=0$, by (3.1), (3.2) and (3.3), we have

$$
\begin{aligned}
& R^{r v x y} \nabla_{x} \nabla_{v} R_{r y}=0, \\
& R^{r v x y} \nabla_{i} R_{r v x y}=0 .
\end{aligned}
$$

On the other hand, in a Riemannian manifold generally we have

$$
\begin{aligned}
\nabla^{h} \nabla_{h}\left(R_{i j k l} R^{i j k l}\right)= & 2\left(\nabla_{h} R_{i j k l} \nabla^{h} R^{i j k l}\right) \\
& +8 R^{i j k l} \nabla_{i} \nabla_{k} R_{j l}+4 R^{i j k l} B_{j k l, h i}^{h},
\end{aligned}
$$

where $B_{j k l, a b}^{i} X^{a} Y^{b}$ are components of $R(X, Y) \cdot R$ (A. Lichnerowicz [2], p. 10). Since (ii) is equivalent to $B_{j k l, h i}^{h}=0$, we have $\nabla_{h} R_{i j k l}=0$.

Proposition 3.2. On $M^{m}(m \geqq 3)$ assume that 
(i) $\nabla R_{1}=0$,

(ii) $(R(X, Y) \cdot R)(X, V)=0$,

(iii) $\left(R(X, Y) \cdot \nabla_{V} R\right)(X, Y)=0$.

Then we have $\nabla R=0$.

Proof. We have (3.1) by (iii). Then we have $\nabla_{h}\left(R_{i j k l} R^{i j k l}\right)=0$. Therefore, (ii) and (3.4) show $\nabla R=0$.

\section{REFERENCES}

1. S. Kobayashi and K. Nomizu, Foundations of differential geometry, Vol. I, WileyInterscience, New York, 1963.

2. A. Lichnerowicz, Géométrie des groupes de transformations, Dunod, Paris, 1958.

3. A. Nijenhuis, On the holonomy groups of linear connections IA, IB, Proc. Kon. Ned. Akad. Amsterdam 15 (1953), 233-249.

4. K. Nomizu, On hypersurfaces satisfying a certain condition on the curvature tensor, Tôhoku Math. J. 20 (1968), 46-59.

5. J. Simons, On the transitivity of holonomy systems, Ann. of Math. 76 (1962), 213234.

6. K. Yano, The theory of Lie derivatives and its applications, Amsterdam, North Holland Pub. Co., 1957.

Received October 6, 1969.

NIIgATA UNIVERSITY

NIIGATA, JAPAN

TOHOKU UNIVERSITY

SENDAI, JAPAN 


\section{PACIFIC JOURNAL OF MATHEMATICS}

EDITORS

H. SAMELSON

Stanford University

Stanford, California 94305

RichaRd PIERCE

University of Washington

Seattle, Washington 98105
J. DUGUNDJI

Department of Mathematics

University of Southern California

Los Angeles, California 90007

RICHARD ARENS

University of California

Los Angeles, California 90024

\section{ASSOCIATE EDITORS}

E. F. BECKENBACH

B. H. NeumanN

F. WOLF

K. YOSHIDA

\section{SUPPORTING INSTITUTIONS}

UNIVERSITY OF BRITISH COLUMBIA

CALIFORNIA INSTITUTE OF TECHNOLOGY

UNIVERSITY OF CALIFORNIA

MONTANA STATE UNIVERSITY

UNIVERSITY OF NEVADA

NEW MEXICO STATE UNIVERSITY

OREGON STATE UNIVERSITY

UNIVERSITY OF OREGON

OSAKA UNIVERSITY

UNIVERSITY OF SOUTHERN CALIFORNIA
STANFORD UNIVERSITY

UNIVERSITY OF TOKYO

UNIVERSITY OF UTAH

WASHINGTON STATE UNIVERSITY

UNIVERSITY OF WASHINGTON

AMERICAN MATHEMATICAL SOCIETY CHEVRON RESEARCH CORPORATION TRW SYSTEMS

NAVAL WEAPONS CENTER 


\section{Pacific Journal of Mathematics}

May, 1970

Johan Aarnes, Edward George Effros and Ole A. Nielsen, Locally compact

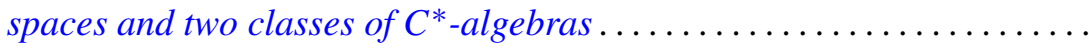

Allan C. Cochran, R. Keown and C. R. Williams, On a class of topological

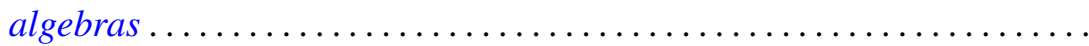

John Dauns, Integral domains that are not embeddable in division rings ....

Robert Jay Daverman, On the number of nonpiercing points in certain

crumpled cubes.....................................

Bryce L. Elkins, Characterization of separable ideals ................

Zbigniew Fiedorowicz, A comparison of two naturally arising uniformities

on a class of pseudo-PM spaces ...........................

Henry Charles Finlayson, Approximation of Wiener integrals of functionals

continuous in the uniform topology ........................

Theodore William Gamelin, Localization of the corona problem ...........

Alfred Gray and Paul Stephen Green, Sphere transitive structures and the

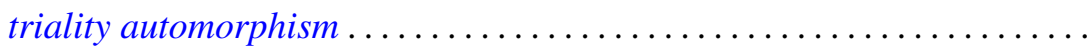

Charles Lemuel Hagopian, On generalized forms of aposyndesis ..........

J. Jakubík, On subgroups of a pseudo lattice ordered group ...............

Cornelius W. Onneweer, On uniform convergence for Walsh-Fourier

series..................................

Stanley Joel Osher, On certain Toeplitz operators in two variables ...

Washek (Vaclav) Frantisek Pfeffer and John Benson Wilbur, On the

measurability of Perron integrable functions............

Frank J. Polansky, On the conformal mapping of variable regions...

Kouei Sekigawa and Shûkichi Tanno, Sufficient conditions for a Riemannian manifold to be locally symmetric ...................

James Wilson Stepp, Locally compact Clifford semigroups ....

Ernest Lester Stitzinger, Frattini subalgebras of a class of solvable Lie

algebras ................................

George Szeto, The group character and split group algebras...

Mark Lawrence Teply, Homological dimension and splitting torsion

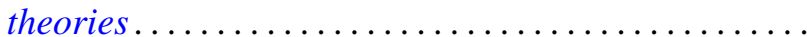

David Bertram Wales, Finite linear groups of degree seven. II ...

Robert Breckenridge Warfield, Jr., An isomorphic refinement theorem for

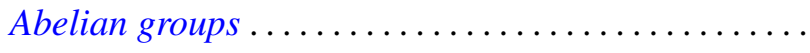

James Edward West, The ambient homeomorphy of an incomplete subspace

of infinite-dimensional Hilbert spaces................

Peter Wilker, Adjoint product and hom functors in general topology ...

Daniel Eliot Wulbert, A note on the characterization of conditional 